\title{
[Eyes are emissaries, soft knocks, nibs]
}

Eyes are emissaries, soft knocks, nibs. Eyes are tongues, mad riverbeds insomniac for salt. Eyes are fangs, bared chisel tattooing face on retina. Bite this word, lick that wound.

Eyes are the itineraries of shooting stars on the tail of new disasters. Faster than witnesses, slow as alibis, don't look! Phoenix of mirages, allusions, holy ash, rising mohair soot. Darkness caving into black diamonds. Lashes fan the air between.

Stones drown to measure water of expression, water nothing dissolves in, pure staring child. Soft convex pillows, seed of sleep.

A false door revolves, a roulette swings back to starting position, the masochists bring out $\mathrm{x}$-ographs.

Unfurling, clitoris. Descriptions, insatiable.

Eyes, are braille. 